Fasting Glucose and the Risk of Depressive Symptoms : Instrumental-Variable Regression in the Cardiovascular Risk in Young Finns Study

\title{
Wesolowska, Karolina
}

2017-12

Wesolowska, K, Elovainio, M , Hintsa , T , Jokela , M , Pulkki-Raback , L, Pitkänen , N , Lipsanen , J , Tukiainen, J , Lyytikäinen, L-P , Lehtimäki , T , Juonala , M , Raitakari , O \& Keltikangas-Järvinen, L 2017 , ' Fasting Glucose and the Risk of Depressive Symptoms : Instrumental-Variable Regression in the Cardiovascular Risk in Young Finns Study ' , International Journal of Behavioral Medicine , vol. 24 , no. 6 , pp. 901-907 . https://doi.org/10.1007/s12529-017-9639

http://hdl.handle.net/10138/298178

https://doi.org/10.1007/s12529-017-9639-2

acceptedVersion

Downloaded from Helda, University of Helsinki institutional repository.

This is an electronic reprint of the original article.

This reprint may differ from the original in pagination and typographic detail.

Please cite the original version. 
Fasting Glucose and the Risk of Depressive Symptoms: Instrumental-Variable Regression in the Cardiovascular Risk in Young Finns Study

Karolina Wesołowska, M.A., Marko Elovainio, Ph.D., Taina Hintsa, Ph.D., Markus Jokela, Ph.D., Laura Pulkki-Råback, Ph.D., Niina Pitkänen, Ph.D., Jari Lipsanen, M.A., Janne Tukiainen, Ph.D., Leo-Pekka Lyytikäinen, M.D., Terho Lehtimäki, M.D.-Ph.D., Markus Juonala, M.D.Ph.D., Olli Raitakari, M.D.-Ph.D., and Liisa Keltikangas-Järvinen, Ph.D.

\author{
University of Helsinki \\ National Institute for Health and Welfare \\ University of Turku \\ VATT Institute for Economic Research \\ University of Tampere \\ Pirkanmaa Hospital District \\ Turku University Hospital \\ Murdoch Children's Research Institute
}

\begin{abstract}
Author Note
Karolina Wesołowska, Taina Hintsa (taina.hintsa@helsinki.fi), Markus Jokela (markus.jokela@helsinki.fi), Jari Lipsanen (jari.lipsanen@helsinki.fi) and Liisa KeltikangasJärvinen (liisa.keltikangas-jarvinen@helsinki.fi), Institute of Behavioral Sciences, University of Helsinki, Helsinki, Finland. Marko Elovainio (marko.elovainio@helsinki.fi), Institute of Behavioral Sciences, University of Helsinki, Helsinki, Finland, and National Institute for Health and Welfare, Helsinki, Finland. Laura Pulkki-Råback (laura.pulkki-raback@helsinki.fi), Institute of Behavioral Sciences and Helsinki Collegium for Advanced Studies, University of Helsinki, Helsinki, Finland. Niina Pitkänen (niina.pitkanen@utu.fi), Research Center of Applied and
\end{abstract}


Preventive Cardiovascular Medicine, University of Turku, Turku, Finland. Janne Tukiainen (janne.tukiainen@vatt.fi), VATT Institute for Economic Research, Helsinki, Finland. Leo-Pekka Lyytikäinen (leo-pekka.lyytikainen@uta.fi) and Terho Lehtimäki (terho.lehtimaki@uta.fi), School of Medicine, University of Tampere, Tampere, Finland, and Department of Clinical Chemistry, Fimlab Laboratories, Pirkanmaa Hospital District, Tampere, Finland. Markus Juonala (markus.juonala@utu.fi), Department of Medicine, University of Turku, Turku, Finland, Division of Medicine, Turku University Hospital, Turku, Finland, and Murdoch Children's Research Institute, Parkville, Victoria, Australia. Olli Raitakari (olli.raitakari@utu.fi), Research Center of Applied and Preventive Cardiovascular Medicine, University of Turku, Turku, Finland, and Department of Clinical Physiology and Nuclear Medicine, Turku University Hospital, Turku, Finland.

Correspondence concerning this article should be addressed to Karolina Wesołowska, Institute of Behavioral Sciences, University of Helsinki, P.O. Box 9 (Siltavuorenpenger 1 A), 00014 Helsinki, Finland. E-mail: karolina.wesolowska@helsinki.fi. Tel.: +358 46 5928090. Fax: +358294129542.

\section{Acknowledgments}

This study was supported by the Academy of Finland: grants 265869 (L. K.-J.), 265977 (M. E.), 286284, 134309, 126925, 121584, 124282, 129378, 117787, and 41071 (T. L.); the Emil Aaltonen Foundation (T. L.); the Finnish Cultural Foundation (T. L.); the Finnish Foundation of Cardiovascular Research (T. L.); the Juho Vainio Foundation (T. L.); the Sigrid Juselius Foundation (N. P.); the Paavo Nurmi Foundation (T. L.); the Yrjö Jahnsson Foundation (T. L.); the Signe and Ane Gylleberg Foundation (L. P.-R.); the Social Insurance Institution of Finland (T. L.); the Tampere Tuberculosis Foundation (T. L.); and the Kuopio, Tampere, and Turku University Hospital Medical Funds (grant X51001, T. L.). 


\begin{abstract}
Purpose: Type 2 diabetes (T2D) has been associated with depressive symptoms, but the causal direction of this association and the underlying mechanisms, such as increased glucose levels, remain unclear. We used instrumental-variable regression with a genetic instrument (Mendelian randomization) to examine a causal role of increased glucose concentrations in the development of depressive symptoms. Methods: Data were from the population-based Cardiovascular Risk in Young Finns Study $(n=1217)$. Depressive symptoms were assessed in 2012 using a modified Beck Depression Inventory (BDI-I). Fasting glucose was measured concurrently with depressive symptoms. A genetic risk score for fasting glucose (with 35 single nucleotide polymorphisms) was used as an instrumental variable for glucose. Results: Glucose was not associated with depressive symptoms in the standard linear regression $(B=-0.04,95 \% C I[-0.12,0.04], p=.34)$, but the instrumental-variable regression showed an inverse association between glucose and depressive symptoms $(B=-0.43,95 \% C I[-0.79,-0.07], p=.020)$. The difference between the estimates of standard linear regression and instrumental-variable regression was significant $(p=$ .026). Conclusions: Our results suggest that the association between T2D and depressive symptoms is unlikely to be caused by increased glucose concentrations. It seems possible that T2D might be linked to depressive symptoms due to low glucose levels.

Keywords: depressive symptoms, fasting glucose, hyperglycemia, instrumental-variable regression, Mendelian randomization, type 2 diabetes (T2D)
\end{abstract}


Two recent meta-analyses of longitudinal studies [1-2] showed that people with type 2 diabetes (T2D) have a 15-24\% higher risk of developing depressive symptoms than those without T2D. It is, however, unclear whether this association is due to the causal effects of pathophysiological processes underlying diabetes [3]. Gaining this knowledge is important as it will have implications for prevention and treatment of these two conditions.

Increased glucose associated with T2D has been investigated as one of the mechanisms linking T2D to depressive symptoms, but the reported results are inconsistent [4-10]. While some studies $[4,6]$ showed a positive association between hyperglycemia and depressive symptoms, others [9-10] suggested that increased glucose may not contribute to depressive symptoms, but rather, that depressive symptoms in T2D may reflect psychosocial stress associated with being diagnosed with T2D, and the burden of coping with T2D and its complications. One study [8] reported an increased risk of depressive symptoms in individuals with very high and very low fasting blood glucose concentrations and some investigations [5, 7] suggested a negative direction of the association.

Determining the role of increased glucose in the development of depressive symptoms has been hampered by methodological limitations of observational data. More advanced methods could improve causal inference. Instrumental-variable regression with a genetic instrument (Mendelian randomization) is a statistical method that can reduce the effects of confounding and reverse causality by using genetic variants as instruments for the exposure of interest, such as glucose levels. Due to the random allocation of alleles during gamete formation, genetic variants are generally independent of any of the environmental factors that typically confound associations between exposures and disease outcomes in non-experimental studies. And as people's genotypes are determined at conception, genetic variants precede health-related outcomes. Thus, instrumental-variable regression with genetic variants as instruments can 
strengthen causal inference by providing estimates that are less likely to be affected by confounding and reverse causality than the estimates derived from conventional multiple regression [11-13]. The assumptions underlying instrumental-variable regression are depicted with more detail in Figure 1.

Insert Figure 1 here

Whereas conventional multiple regression examines how the outcome is related to the total variation in the risk factor across individuals, instrumental-variable regression is based only on the proportion of variation in the risk factor that is associated with the genetic variants used as the instrument for the risk factor. If the estimates of conventional multiple regression are biased by confounding or reverse causality, one would expect these estimates to differ from the estimates of instrumental-variable regression. If, on the other hand, the estimates derived from conventional regression do not differ significantly from the estimates of instrumental-variable regression, the risk factor can be assumed to be exogenous, in which case the estimates of the conventional regression model are preferable because they are more efficient, that is, more precise [14].

In this study, we implemented instrumental-variable regression with a genetic risk score for fasting glucose used as a proxy for fasting glucose to test whether increased glucose is causally associated with depressive symptoms. 


\section{Methods}

\section{Participants}

The participants were from the Cardiovascular Risk in Young Finns Study, an ongoing, population-based prospective study examining cardiovascular risk factors in Finland [15-16]. The study has been conducted in all Finnish cities with a medical school (Helsinki, Kuopio, Oulu, Tampere, and Turku) and their rural surroundings. In total, 4320 healthy white Finnish children and adolescents aged 3, 6, 9, 12, 15, and 18 years were randomly selected using their unique personal identification numbers. Of these individuals, $3596(83.2 \%)$ participated at the baseline study in 1980. Since that time, eight follow-up studies have been performed in: 1983, 1986, 1989, 1992, 1997, 2001, 2007, and 2012. In the latest data collection phase, there were 2063

participants (45\% men). So far, 104 individuals have died and 1429 individuals have dropped out [17]. The study was approved by the local ethics committees. All the participants gave their written informed consent.

The present cross-sectional study was based on data from the 2012 follow-up. Of the 2063 individuals, $796(38.6 \%)$ had some missing information on the study variables. People with the diagnosis of type 1 diabetes (T1D; $n=7)$ and T2D $(n=43)$ were excluded. Thus, the final sample consisted of 1217 participants.

\section{Measures}

Depressive symptoms in 2012 were self-rated with a modified version of the Beck Depression Inventory-I (BDI-I) [18], which was translated into Finnish and adopted for use in the Cardiovascular Risk in Young Finns Study [19-20]. The original version of the measure has 21 items with four alternative response statements in each item ( 0 indicating absence and 1-3 indicating increasing levels in the severity of depressive symptoms). In the modified version, the items are the second mildest statements of the BDI-I items, selected because they were suggested 
to capture a wider variation in depressive symptoms in the general population compared with the original version of the measure [21]. The participants were asked to rate each of the 21 items on a 5-point scale ranging from totally disagree (1) to totally agree (5). We calculated depressive symptoms' severity score for each participant as the mean score of responses instead of classifying the participants into depressive symptoms' groups. This was done due to two reasons: first, there have been no standardized cut-off points for the modified BDI-I and data-driven cutoff points create a difficulty to compare results across different studies [22], and second, categorization of continuous variables may be associated with a loss of statistical power and an imprecise estimation [22]. The scale reliability (Cronbach's $\alpha$ ) was .92 . The modified BDI-I has been shown to correlate with $R=.77(p<.001)$ with the BDI-II which is a tool to screen for clinical depression [21]. The validity of the modified BDI-I is also supported by previous studies that have associated this instrument with psychosocial characteristics known to be associated with depressive symptoms, such as negative emotionality and low sociability [23], fatigability and sentimentality [24], and hostility and low social support [25]. Additionally, the measure has been shown to be a valid predictor of risk factors for cardiovascular disease, such as preclinical atherosclerosis [26] and the metabolic syndrome [27].

Fasting serum glucose concentration was measured in 2012 with the enzymatic hexokinase method (Glucose reagent, Beckman Coulter Biomedical) using an AU400 instrument (Olympus, Japan). Body mass index (BMI) in 2012 was calculated as weight $(\mathrm{kg}) /$ height $^{2}\left(\mathrm{~m}^{2}\right)$. Height was measured with a wall-stated stadiometer and weight with Seca scales. Physical activity in 2012 was measured with a questionnaire consisting of five items concerning: the intensity of physical activity, frequency of vigorous physical activity, hours spent on vigorous physical activity, average duration of a physical activity session, and participation in organized physical activity. 
The scale was previously described in detail by Telama et al. [28]. Overall physical activity was calculated as the sum score of responses.

Both BMI and physical activity have been associated with T2D and depressive symptoms [3]. Therefore, the potential confounding effects of the two variables, together with the effects of sex and age, were controlled in our analyses.

The genome wide single nucleotide polymorphism (SNP) analyses (GWAS) for the Cardiovascular Risk in Young Finns Study participants were performed in 2009 by using the 670K Illumina platform (Sanger Institute, UK). Variation in over 670000 known SNPs was measured from 2627 participants. Genotype imputation was performed using SHAPEIT [29] and IMPUTE2 software [30], and the 1000G Phase I Integrated Release Version 3 Haplotypes [31] as a reference panel. A weighted genetic risk score comprising 35 previously published risk SNPs for fasting glucose [32-34] was calculated as the sum of genotyped risk alleles or imputed allele dosages carried by an individual, each multiplied by the effect size (the natural log of the odds ratios) using R software version 2.15.3. Details of these SNPs are provided in Supplementary Table 1. We used a weighted instead of an unweighted genetic risk score as the use of a weighted allele score has been shown to increase statistical power [35].

\section{Statistical Analysis}

The statistical analysis was performed using Stata/SE 13 software. The association between glucose and depressive symptoms was examined with standard linear regression and instrumental-variable regression (the generalized method of moments, GMM) [36] controlling for sex, age, BMI and physical activity, and allowing for heteroscedasticity of the error term by using "wmatrix (robust)" option [36]. The results of the standard linear regression and the instrumentalvariable regression were compared implementing the difference-in-Sargan test (the $C$ statistic) [36]. The associations between the genetic risk score and the study covariates were tested with 
linear and binary logistic regression. The strength of the association of the genetic risk score with glucose was evaluated on the basis of the $F$-statistic from the first-stage regression analysis. The $F$-value greater than 10 is commonly used as evidence that an instrument is sufficiently strong [37]. The attrition analysis was carried out with the two-sample $t$-test and the chi-square test.

Additionally, the main analysis was repeated in the imputed data for all of those participants of the Young Finns Study who had information on the genetic risk score for fasting glucose and who did not have the diagnosis of T1D or T2D in $2012(n=2527)$. Multiple imputed values were generated for the missing data from the variables used in the analysis with Stata imputation by chained equations (ice) [38]. Fifty datasets were randomly selected and then analyzed using "mi estimate" option and "mibeta" command.

\section{Results}

Individuals included in the study sample $(n=1217)$ and those who were lost due to attrition did not differ in age (42.91 vs. $42.61, p=.19)$, the genetic risk score for fasting glucose $(-0.04$ vs. $-0.001, p=.41)$, and physical activity (9.05 vs. $8.93, p=.22)$. The groups differed, however, in respect of depressive symptoms (2.04 vs. 2.22, $p<.001)$, glucose levels (5.26 vs. 5.42, $p<$ $.001)$, and BMI (26.07 vs. 26.94, $p<.001)$. Also, more men than women dropped out $(45.4 \%$ vs. $33.9 \%, p<.001)$. Table 1 summarizes the characteristics of the study participants.

Insert Table 1 here

Table 2 shows the associations between the genetic risk score and the study covariates. The genetic risk score was not associated with sex, age, BMI, or physical activity. There was an 
association of the genetic risk score with glucose $(B=0.09,95 \% C I[0.07,0.12], p<.001)$ and depressive symptoms $(B=-0.0495 \% C I[-0.07,-0.005], p=.025)$. The $F$-value of the association of the genetic risk score with glucose while adjusting for sex, age, BMI, and physical activity indicated that the genetic risk score was a sufficiently strong genetic instrument for this variable $\left(F=69.72, R^{2}=22.35 \%\right)$.

Insert Table 2 here

The results of the standard linear regression and the instrumental-variable regression with their comparison are shown in Table 3. In the standard linear regression analysis, depressive symptoms were not associated with glucose $(B=-0.04,95 \% C I[-0.12,0.04], p=.34)$ when controlling for sex, age, BMI, and physical activity. In the instrumental-variable regression, however, the effect of glucose on depressive symptoms was statistically significant after adjustment for those four covariates $(B=-0.43,95 \% C I[-0.79,-0.07], p=.020)$. The difference between the estimates of standard linear regression and instrumental-variable regression models was also significant $(p=.026)$.

Insert Table 3 here 
The results of the analysis performed in the data with imputed values are presented in Supplementary Table 2 and Supplementary Table 3. The assumptions underlying instrumentalvariable regression were met. The strength of the association between the genetic risk score and glucose was sufficient $\left(F=98.20 ; R^{2}=21.83 \%\right)$. The results of the standard-linear regression showed that glucose was not associated with depressive symptoms after controlling for sex, age, BMI, and physical activity $(B=-0.02,95 \% C I[-0.11,0.06], p=.58)$. In the fully adjusted instrumental-variable regression model, the association between glucose and depressive symptoms was, however, statistically significant $(B=-0.42,95 \% C I[-0.76,-0.08], p=.015)$. The difference between the estimates of standard linear regression and instrumental-variable regression models were significant in 48 out of 50 imputed datasets $(p s<.05)$. Overall, these additional tests suggest that it is unlikely that the results derived from the analysis of the incomplete data could be biased by the attrition.

\section{Discussion}

To our knowledge, this is the first study in which the Mendelian randomization approach was applied to examine a causal role of increased glucose in the development of depressive symptoms. The results of the standard linear regression showed that glucose is not associated with depressive symptoms when controlling for sex, age, BMI, and physical activity. The instrumental-variable regression showed a negative association between glucose and depressive symptoms in the fully adjusted model. The difference between the estimates derived from the two methods was significant. Thus, these results do not support increased glucose levels to be a causal risk factor for depressive symptoms in people with T2D. The association between T2D and depressive symptoms might be due to hypoglycemia. 
Our finding is in line with the results of two observational studies [5,7], which suggested that the association between hyperglycemia and depressive symptoms may be negative. Insulin resistance related to $\mathrm{T} 2 \mathrm{D}$ has also been inversely associated with the risk of depressive symptoms (together with depression) [39-40], although not in all studies [41-43]. The present study, however, advances previous investigations about the association between glucose levels and depressive symptoms [4-10] by utilizing genetic information in instrumental-variable regression to reduce bias caused by confounding and reverse causation, and thus to strengthen causal inference regarding this association. Our results support the conclusion of a recent literature review [3] of observational studies that the pathophysiology of T2D, such as chronic hyperglycemia, is unlikely to contribute to depressive symptoms. Our results suggest that the association between T2D and depressive symptoms could be mediated by low glucose concentrations. There is some evidence that hypoglycemia, affecting approximately $23 \%$ of individuals with T2D diagnosis [44], is associated with an increased risk of depressive symptoms in this group of patients [44-45].

One of the assumptions underlying instrumental-variable regression is that the genetic variants used as an instrumental variable should influence the outcome only through the exposure of interest [13]. In practice, this methodological assumption is untestable [46-47] and it can be violated by, for instance, population stratification and pleiotropy [13]. It is unlikely that our results were affected by population stratification as the study sample consisted of only native Finnish people. Pleiotropic genetic effects might violate the assumption if the genetic instrument directly influenced the outcome or if the association between the genetic instrument and the outcome was mediated by other factors besides the exposure of interest. It is possible that the 35 SNP genetic risk score for fasting glucose influences depressive symptoms via other pathways besides glucose. If that was proven to be true, the above assumption underlying instrumental- 
variable regression would be violated, and thus the causal link between glucose and depressive symptoms would be overestimated.

Depressive symptoms were self-reported with a modified version of the BDI-I which is not a measure of clinical depression but a measure of mild depressive symptoms. Glucose concentrations might be related to depressive symptoms differently than to depression [4]. Thus, our results may not be applicable to clinically diagnosed depression.

However, depressive symptoms may also be considered as an indicator of an early stage of clinical depression. Subthreshold depressive symptoms have been shown to predict an increased risk of developing major depressive disorder later on [48]. Decreased glucose could thereby increase the risk of clinical depression by contributing to the progression of depressive symptoms.

A relatively large population-based sample provided a methodological strength to our study. The inclusion of a young cohort (an age range of 35 to 50 years) can be seen as both a strength and a limitation. Due to a paucity of studies in the field of T2D and depressive symptoms conducted on younger populations, our study makes a unique contribution in this respect. However, the age range may have underestimated the role of glucose in the development of depressive symptoms in older individuals, given that the incidence of T2D begins to increase rapidly after midlife [49].

In conclusion, our results provide evidence suggesting that it is unlikely that the association between T2D and depressive symptoms is caused by increased glucose concentrations. Furthermore, it seems possible that T2D might be linked to depressive symptoms due to low glucose levels. Considering the methodological limitations of instrumental-variable regression, this finding should be interpreted carefully and needs to be replicated in other studies. In addition, we suggest that in further studies more attention could be given to possible mediating factors of 
the association between the genetic risk score for fasting glucose and depressive symptoms. Moreover, future research needs to focus on testing the association of glucose with clinically diagnosed depression. Additional studies examining this association in older populations, among whom T2D is more prevalent, are also of value. Finally, the present results suggest that the effect of depressive symptoms on the development of T2D requires further examination, especially using genetic data.

\section{Compliance with Ethical Standards}

\section{Conflicts of Interest}

The authors declare that they have no conflict of interest.

Human and Animal Rights

All procedures performed in studies involving human participants were in accordance with the ethical standards of the institutional and/or national research committee and with the 1964 Helsinki declaration and its later amendments or comparable ethical standards. This article does not contain any studies with animals performed by any of the authors.

\section{Informed Consent}

Informed consent was obtained from all individual participants included in the study. 


\section{References}

1. Mezuk B, Eaton WW, Albrecht S, Golden SH. Depression and type 2 diabetes over the lifespan: a meta-analysis. Diabetes Care. 2008;31(12):2383-90.

2. Nouwen A, Winkley K, Twisk $\mathbf{J}$, et al. Type 2 diabetes mellitus as a risk factor for the onset of depression: a systematic review and meta-analysis. Diabetologia. 2010;53(12):2480-6.

3. Tabák AG, Akbaraly TN, Batty GD, Kivimäki M. Depression and type 2 diabetes: a causal association? The Lancet Diabetes and Endocrinology. 2014;2(3):236-45.

4. Gale CR, Kivimaki M, Lawlor DA, Carroll D, Phillips AC, Batty GD. Fasting glucose, diagnosis of type 2 diabetes, and depression: The Vietnam Experience Study. Biol Psychiat. 2010;67(2):189-92.

5. Golden SH, Lazo M, Carnethon M, et al. Examining a bidirectional association between depression and diabetes. J Amer Med Assoc. 2008;299(23):2751-9.

6. Hamer M, Batty GD, Kivimaki M. Haemoglobin A1c, fasting glucose and future risk of elevated depressive symptoms over 2 years of follow-up in the English Longitudinal Study of Ageing. Psychol Med. 2011;41(9):1889-96.

7. Icks A, Kruse J, Dragano N, et al. Are symptoms of depression more common in diabetes? Results from the Heinz Nixdorf Recall study. Diabetic Med. 2008;25(11):1330-6.

8. Kivimaki M, Tabak AG, Batty GD, et al. Hyperglycemia, type 2 diabetes, and depressive symptoms: The British Whitehall II study. Diabetes Care. 2009;32(10):1867-9.

9. Mezuk B, Johnson-Lawrence V, Lee H, et al. Is ignorance bliss? Depression, antidepressants, and the diagnosis of prediabetes and type 2 diabetes. Health Psychol. 2013;32(3):254-63. 
10. Nouwen A, Nefs G, Caramlau I, et al. Prevalence of depression in individuals with impaired glucose metabolism or undiagnosed diabetes: a systematic review and meta-analysis of the European Depression in Diabetes (EDID) Research Consortium. Diabetes Care. 2011;34(3):752-62.

11. Davey Smith G, Ebrahim S. Mendelian randomization: prospects, potentials, and limitations. Int J Epidemiol. 2004;33(1):30-42.

12. Davey Smith G, Lawlor DA, Harbord R, Timpson N, Day I, Ebrahim S. Clustered environments and randomized genes: a fundamental distinction between conventional and genetic epidemiology. PLOS Med. 2007;4(12):e352.

13. Lawlor DA, Harbord RM, Sterne JA, Timpson N, Davey Smith G. Mendelian randomization: using genes as instruments for making causal inferences in epidemiology. Stat Med. 2008;27(8):1133-63.

14. Baum CF. An introduction to modern econometrics using Stata. College Station, Texas: Stata Press; 2006.

15. Akerblom HK, Uhari M, Pesonen E, et al. Cardiovascular risk in young Finns. Ann Med. 1991;23(1):35-9.

16. Raitakari OT, Juonala M, Rönnemaa T, et al. Cohort profile: The Cardiovascular Risk in Young Finns Study. Int J Epidemiol. 2008;37(6):1220-6.

17. Nuotio J, Oikonen M, Magnussen CG, et al. Cardiovascular risk factors in 2011 and secular trends since 2007: The Cardiovascular Risk in Young Finns Study. Scand J Public Healt. 2014;42(7):563-71. 
18. Beck AT, Steer RA. Manual for the Beck Depression Inventory. San Antonio, TX: Psychological Corporation; 1987.

19. Elovainio M, Jokela M, Rosenström T, et al. Temperament and depressive symptoms: what is the direction of the association? J Affect Disorders. 2015;170:203-12.

20. Katainen S, Raikkonen K, Keskivaara P, Keltikangas-Jarvinen L. Maternal child-rearing attitudes and role satisfaction and children's temperament as antecedents of adolescent depressive tendencies follow-up study of 6- to 15-year-olds. J Youth Adolescence. 1999;28(2):139-63.

21. Rosenstrom T, Jokela M, Puttonen S, et al. Pairwise measure of causal direction in the epidemiology of sleep problems and depression. PLOS One. 2012;7(11):e50841.

22. Bennette C, Vickers A. Against quantiles: categorization of continuous variables in epidemiologic research, and its discontents. BMC Med Res Methodol. 2012; doi:10.1186/1471-2288-12-21

23. Katainen S, Raikkonen K, Keltikangas-Jarvinen L. Adolescent temperament, perceived social support, and depressive tendencies as predictors of depressive tendencies in young adulthood. Eur J Personality. 1999;13(3):183-207.

24. Elovainio M, Kivimaki M, Puttonen S, Heponiemi T, Pulkki L, Keltikangas-Jarvinen L. Temperament and depressive symptoms: a population-based longitudinal study on Cloninger's psychobiological temperament model. J Affect Disorders. 2004;83(2-3):227-32.

25. Heponiemi T, Elovainio M, Kivimaki M, Pulkki L, Puttonen S, Keltikangas-Jarvinen L. The longitudinal effects of social support and hostility on depressive tendencies. Soc Sci Med. 2006;63(5):1374-82. 
26. Elovainio M, Keltikangas-Jarvinen L, Kivimaki M, et al. Depressive symptoms and carotid artery intima-media thickness in young adults: The Cardiovascular Risk in Young Finns Study. Psychosom Med. 2005;67(4):561-7.

27. Pulkki-Raback L, Elovainio M, Kivimaki M, et al. Depressive symptoms and the metabolic syndrome in childhood and adulthood. Health Psychol. 2009;28(1):108-116.

28. Telama R, Yang X, Viikari J, Välimäki I, Wanne O, Raitakari O. Physical activity from childhood to adulthood: a 21-year tracking study. Am J Prev Med. 2005;28(3):267-73.

29. Delaneau O, Marchini J, Zagury JF. A linear complexity phasing method for thousands of genomes. Nat Methods. 2011;9(2):179-81.

30. Howie BN, Donnelly P, Marchini J. A flexible and accurate genotype imputation method for the next generation of genome-wide association studies. PLOS Genet. 2009;5(6):e1000529.

31. The 1000 Genomes Project Consortium. A map of human genome variation from population scale sequencing. Nature. 2010;467(7319):1061-73.

32. Dupuis J, Langenberg C, Prokopenko I, et al. New genetic loci implicated in fasting glucose homeostasis and their impact on type 2 diabetes risk. Nat Genet. 2010;42(2):105-16.

33. Manning AK, Hivert MF, Scott RA, et al. A genome-wide approach accounting for body mass index identifies genetic variants influencing fasting glycemic traits and insulin resistance. Nat Genet. 2012;44(6):659-69.

34. Scott RA, Lagou V, Welch RP, et al. Large-scale association analyses identify new loci influencing glycemic traits and provide insight into the underlying biological pathways. Nat Genet. 2012;44(9):991-1005. 
35. Burgess $\mathrm{S}$, Thompson SG. Use of allele scores as instrumental variables for Mendelian randomization. Int J Epidemiol. 2013;42(4):1134-44.

36. Baum CF, Schaffer ME, Stillman S. Instrumental variables and GMM: estimation and testing. Stata J. 2003;3(1):1-31.

37. Staiger D, Stock JH. Instrumental variables regression with weak instruments. Econometrica. 1997;65(3):557-86.

38. Royston P, White IR. Multiple imputation by chained equations (MICE): implementation in Stata. J Stat Softw. 2011;45(4):1-20.

39. Golomb BA, Tenkanen L, Alikoski T, et al. Insulin sensitivity markers: predictors of accidents and suicides in Helsinki Heart Study screenees. J Clin Epidemiol. 2002;55(8):76773.

40. Lawlor DA, Davey Smith G, Ebrahim S. Association of insulin resistance with depression: cross-sectional findings from the British Women's Heart and Health Study. Brit Med J. 2003;327(7428):1383-4.

41. Lawlor DA, Ben-Shlomo Y, Ebrahim S, Davey Smith G. Insulin resistance and depressive symptoms in middle aged men: findings from the Caerphilly prospective cohort study. Brit Med J. 2005;330(7493):705-6.

42. Pearson S, Schmidt M, Patton G, et al. Depression and insulin resistance: cross-sectional associations in young adults. Diabetes Care. 2010;33(5):1128-33.

43. Timonen M, Laakso M, Jokelainen J, Rajala U, Meyer-Rochow VB, KeinanenKiukaanniemi S. Insulin resistance and depression: cross sectional study. Brit Med J. $2005 ; 330(7481): 17-8$. 
44. Green AJ, Fox KM, Grandy S. Self.reported hypoglycemia and impact on quality of life and depression among adults with type 2 diabetes mellitus. Diabetes Res Clin Pr. 2012;96(3):313-8.

45. Kikuchi Y, Iwase M, Fujii H, et al. Association of severe hypoglycemia with depressive symptoms in patients with type 2 diabetes: The Fukuoka Diabetes Registry. BMJ Open Diabetes Res Care. 2015;3(1):e000063.

46. Glymour MM, Tchetgen Tchetgen EJ, Robins JM. Credible Mendelian randomization studies: approaches for evaluating the instrumental variable assumptions. Am J Epidemiol. 2012;175(4):332-9.

47. Morgan SL, Winship C. Counterfactuals and causal inference: methods and principles for social research. 1st ed. New York: Cambridge University Press; 2007.

48. Karsten J, Hartman CA, Ormel J, Nolen WA, Penninx BW. Subthreshold depression based on functional impairment better defined by symptom severity than by number of DSM-IV symptoms. J Affect Disorders. 2010;123(1-3):230-7.

49. Wild S, Roglic G, Green A, Sicree R, King H. Global prevalence of diabetes: estimates for the year 2000 and projections for 2030. Diabetes Care. 2004;27(5):1047-53. 


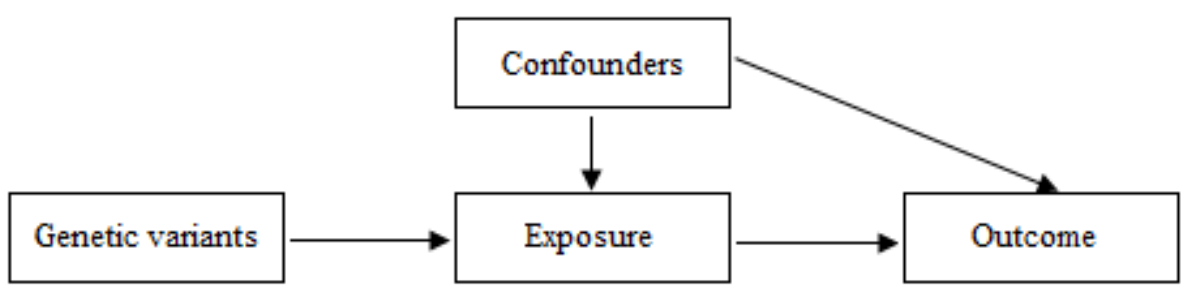

Figure 1. Assumptions underlying instrumental-variable regression. Genetic variants can be used as an instrumental variable for a modifiable exposure if they are: 1) associated with the exposure of interest; 2) independent of any possible factors confounding the association between the exposure and the outcome; and 3) associated with the outcome only through the exposure of interest. 
Table 1

Descriptive Statistics $(n=1217)$

\begin{tabular}{lccc}
\hline Study variables & $n(\%)$ & $M(S D)$ & Range \\
\hline Sex & 1217 & & \\
$\quad$ Men & & & \\
Women & $717(58.9)$ & & \\
Age (years) & & $42.91(5.00)$ & $35.00-50.00$ \\
Depressive symptoms & & $2.04(0.62)$ & $1.00-4.43$ \\
35-SNP genetic risk score & & $-0.04(1.01)$ & $-3.28-3.01$ \\
Glucose (mmol/L) & & $5.26(0.48)$ & $3.14-6.84$ \\
BMI (kg/m $\left.{ }^{2}\right)$ & & $26.07(4.67)$ & $17.01-58.47$ \\
Physical activity & & $9.05(1.85)$ & $5.00-15.00$
\end{tabular}

Abbreviations: $M$, mean; $n$, number of participants; $S D$, standard deviation. The 35-SNP genetic risk score expressed in standard score. 
Table 2

Associations Between the 35-SNP Genetic Risk Score of Fasting Glucose and the Study

Covariates $(n=1217)$

\begin{tabular}{lcccc}
\hline & \multicolumn{5}{c}{ Genetic risk score } \\
\cline { 2 - 5 } Outcome & $B$ & $95 \% C I$ & $R^{2}(\%)$ & $p$-value \\
\hline & & & & \\
Sex & 0.002 & {$[-0.11,0.12]$} & 0.00 & .97 \\
Age & 0.03 & {$[-0.25,0.31]$} & 0.00 & .83 \\
BMI & -0.14 & {$[-0.40,0.12]$} & 0.09 & .30 \\
Physical activity & -0.08 & {$[-0.18,0.02]$} & 0.19 & .13 \\
Glucose & 0.09 & {$[0.07,0.12]$} & 3.84 & $<.001$ \\
Depressive symptoms & -0.04 & {$[-0.07,-0.005]$} & 0.41 & .025
\end{tabular}

Abbreviations: $B$, unstandardized linear regression coefficient; $C I$, confidence interval; $R^{2}$, fraction of explained variance (\%). 
Table 3

Standard Linear Regression and Instrumental-Variable Regression Predicting Depressive Symptoms With Glucose $(n=1217)$

\begin{tabular}{|c|c|c|c|c|c|c|c|}
\hline \multirow[b]{2}{*}{ Predictors } & \multicolumn{3}{|c|}{ Standard linear regression } & \multicolumn{3}{|c|}{ Instrumental-variable regression $^{\mathrm{a}}$} & \\
\hline & $B$ & $95 \% C I$ & $p$-value & $B$ & $95 \% C I$ & $p$-value & $\begin{array}{c}p \text {-value for a difference } \\
\text { between the models }^{\mathrm{b}}\end{array}$ \\
\hline Glucose & -0.04 & {$[-0.12,0.04]$} & .34 & -0.43 & {$[-0.79,-0.07]$} & .020 & .026 \\
\hline
\end{tabular}

\footnotetext{
Abbreviations: $B$, unstandardized linear regression coefficient; $C I$, confidence interval.

Models adjusted for sex, age, BMI, and physical activity.

${ }^{a}$ In the instrumental-variable regression, the 35-SNP genetic risk score of fasting glucose was used as an instrumental variable for glucose.

${ }^{b}$ Difference-in-Sargan test.
} 
Supplementary Table 1

Details of the 35 SNPs Used to Calculate the Genetic Risk Score for Fasting Glucose

\begin{tabular}{|c|c|c|}
\hline SNP & Nearby gene $(s)^{\mathrm{a}}$ & Minor allele frequency ${ }^{b}$ \\
\hline rs 560887 & G6PC2 & .31 \\
\hline rs 10830963 & $M T N R 1 B$ & .36 \\
\hline rs4607517 & $G C K$ & .11 \\
\hline rs2191349 & $D G K B-T M E M 195$ & .46 \\
\hline rs 780094 & $G C K R$ & .36 \\
\hline rs 10811661 & $C D K N 2 B$ & .15 \\
\hline rs3736594 & MRPL33 & .33 \\
\hline rs4869272 & PCSK1 & .33 \\
\hline rs983309 & $P P P 1 R 3 B$ & .18 \\
\hline rs11708067 & $A D C Y 5$ & .18 \\
\hline rs1371614 & DPSYL5 & .23 \\
\hline rs6943153 & GRB10 & .49 \\
\hline rs11603334 & $A R A P 1$ & .26 \\
\hline rs6048205 & FOXA2 & .05 \\
\hline rs16913693 & $I K B K A P$ & .02 \\
\hline rs2293941 & $P D X 1$ & .26 \\
\hline rs7944584 & $M A D D$ & .19 \\
\hline rs3829109 & $D N L Z$ & .36 \\
\hline rs3783347 & WARS & .19 \\
\hline rs13179048 & PCSK1 & .33 \\
\hline rs 13266634 & SLC $30 A 8$ & .38 \\
\hline rs2302593 & $G I P R$ & .48 \\
\hline rs9368222 & CDKAL1 & .34 \\
\hline rs7034200 & GLIS3 & .50 \\
\hline rs 10747083 & $P 2 R X 2$ & .38 \\
\hline rs 174550 & FADS1 & .40 \\
\hline rs 17762454 & RREB1 & .29 \\
\hline rs7708285 & ZBED3 & .22 \\
\hline rs6072275 & TOP1 & .24 \\
\hline rs7651090 & $I G F 2 B P 2$ & .31 \\
\hline rs576674 & $K L$ & .10 \\
\hline rs7903146 & TCF $7 L 2$ & .19 \\
\hline rs1483121 & OR4S1 & .12 \\
\hline rs2657879 & $G L S 2$ & .19 \\
\hline rs11715915 & $A M T$ & .44 \\
\hline
\end{tabular}

${ }^{\mathrm{a} A l l}$ information derived from Dupuis et al. [32], Manning et al. [33], and Scott et al. [34].

bata calculated based on the Cardiovascular Risk in Young Finns Study sample. 
Supplementary Table 2

Associations Between the 35-SNP Genetic Risk Score of Fasting Glucose and the Study Covariates in the Data With Imputed Values $(n=2527)$

\begin{tabular}{lcccc}
\hline \multirow{2}{*}{ Outcome } & \multicolumn{5}{c}{ Genetic risk score } \\
\cline { 2 - 5 } & $B$ & $95 \%$ CI & $R^{2}(\%)$ & $p$-value \\
\hline \multirow{2}{*}{ Sex } & -0.02 & {$[-0.10,0.06]$} & 0.01 & .63 \\
Age & -0.17 & {$[-0.36,0.03]$} & 0.12 & .088 \\
BMI & -0.20 & {$[-0.42,0.01]$} & 0.20 & .068 \\
Physical activity & -0.03 & {$[-0.12,0.06]$} & 0.05 & .53 \\
Glucose & 0.09 & {$[0.07,0.11]$} & 3.54 & $<.001$ \\
Depressive symptoms & -0.04 & {$[-0.08,-0.01]$} & 0.48 & .012
\end{tabular}

Abbreviations: $B$, unstandardized linear regression coefficient; $C I$, confidence interval; $R^{2}$, fraction of explained variance (\%). 
Supplementary Table 3

Standard Linear Regression and Instrumental-Variable Regression Predicting Depressive

Symptoms With Glucose in the Data With Imputed Values $(n=2527)$

\begin{tabular}{|c|c|c|c|c|c|c|}
\hline \multirow[b]{2}{*}{ Predictors } & \multicolumn{3}{|c|}{ Standard linear regression } & \multicolumn{3}{|c|}{ Instrumental-variable regression } \\
\hline & $B$ & $95 \% C I$ & $p$-value & $B$ & $95 \% C I$ & $p$-value \\
\hline Glucose & -0.02 & {$[-0.11,0.06]$} & .58 & -0.42 & {$[-0.76,-0.08]$} & .015 \\
\hline
\end{tabular}

Abbreviations: $B$, unstandardized linear regression coefficient; $C I$, confidence interval.

Models adjusted for sex, age, BMI, and physical activity.

In the instrumental-variable regression, the 35-SNP genetic risk score of fasting glucose was used as an instrumental variable for glucose. 